\title{
Prevalence and risk factors of diabetes mellitus among adults in Jaffna District
}

\author{
S Amarasinghe, S Balakumar, V Arasaratnam \\ (Index words: diabetes mellitus, prevalence, risk factors)
}

\begin{abstract}
A cross sectional descriptive study was carried out to determine the prevalence and risk factors of diabetes mellitus among adults in Jaffna District. Multistage stratified cluster sampling technique was employed to select 544 participants. An interviewer administrated questionnaire was used. Anthropometric and blood pressure (BP) measurements were recorded and biochemical parameters were analysed. Response rate was $95.3 \%$. Of them, $224(43.8 \%)$ were male. The prevalence of diabetes mellitus was $16.4 \%$ (95\% Cl: $13.3-$ 19.9); in males $19.6 \%$ (95\% Cl: 14.6-25.4) and in females $13.9 \%$ (95\% Cl: 10.1-18.5). Of the diabetics, $27.4 \%$ were previously undiagnosed. In the final multivariable model, participants with family history of diabetes were 3.5 times $(p<0.001)$ more likely and those with high waist hip ratio were 2 times $(p=0.009)$ more likely to develop diabetes mellitus.
\end{abstract}

Ceylon Medical Journal 2015; 60: 107-110

\section{Introduction}

Epidemiologic studies performed in South Asian countries have demonstrated a progressive and alarming increase in the incidence of cardiovascular disease together with a increase in the prevalence of type 2 diabetes [1]. None of the previous studies in Sri Lanka covered the population in Jaffna because of the difficulty in accessing the area during the civil war. Our aim was to determine the prevalence and risk factors of diabetes mellitus (DM) among adults in Jaffna District.

\section{Methods}

A cross sectional community based descriptive study in Jaffna District was conducted among people above 18 years. Multistage stratified cluster sampling was employed.

Population in the Jaffna district was stratified into urban and rural sectors. Thirty two clusters were selected after considering feasibility and wide scattered spread of sample. Based on the proportions in the population 25 clusters were selected from the rural (79.8\% of population) and 7 clusters were selected from the urban (20.2\% of population) areas [2]. A Grama Niladari division (GND) was considered as a cluster. A household was randomly selected from each cluster. The randomly selected house was located and visited as the first house. The one closest to the right side of the front door of the first house was visited next. This procedure was repeated until the required number of respondents were interviewed in each cluster. In each house visited, all eligible males and females were listed and the person to be interviewed was selected randomly using the lottery method. The prevalence of DM (18.7\%) from a preliminary study was used to calculate the sample size. Confidence interval of $95 \%$ and margin of error of 5\% were considered acceptable [3].

Sample size was calculated using the formula $\mathrm{n}=\mathrm{z}^{2} \times \mathrm{P}(100-\mathrm{P}) / \mathrm{d}^{2}$

Calculated sample size was 234. The design effect for this particular population study was unknown. Thus, the design effect was considered as two. Calculated sample size after applying design effect was 468 . Non response rate was assumed as $10 \%$. Thus, sample size after correction for non responders was 515 . This figure was rounded to 544 considering number of clusters. Approval was obtained from the Ethics Review Committee, Faculty of Medicine, University of Jaffna. Informed written consent was obtained from each participant.

Body weight was measured with light clothes without shoes to the nearest $100 \mathrm{~g}$ using an electronic digital weighing scale. Height was measured using a stadiometer without shoes with the participant looking straight ahead. Waist circumference (WC) was measured by positioning the non elastic measuring tape midway between the lower rib margin and the iliac crest, at the end of a normal expiration. Hip circumference (HC) was measured with a non elastic measuring tape at maximal circumference at the buttocks. Blood pressure (BP) was measured in the seated position after the participants had rested for at least 5 minutes. The measurement was taken using the supported

Department of Biochemistry, Faculty of Medicine, University of Jaffna, Sri Lanka.

Correspondence: AS, e-mail: <rathybio@gmail.com>. Received 17 April and revised version accepted 5 July 2015. 
left arm at the heart level, using a sphygmomanometer. Two recordings were taken and the mean was used for analysis. In the event of variation of over $20 \mathrm{mmHg}$ between recordings, a third reading was done and the mean of the last two recordings was used [4]. Overnight fasting blood samples were obtained from all participants. An interviewer administrated questionnaire was employed to collect relevant data.

GN divisions in the Jaffna Municipal Council and Urban Councils areas were considered as urban sector. GN divisions in the Pradesheeya Sabha areas were considered as rural sector [2]. Age was considered as a categorical variable. Physical activity level was classified into three categories - insufficiently active (no activity is reported /some activity is reported but not enough to meet categories of sufficiently active or highly active), sufficiently active ( $\geq 600$ Metabolic Equivalent of Task (MET)-minutes/week) and highly active ( $\geq 3,000$ METminutes/ week) by using the International Physical Activity questionnaire.

Fasting plasma glucose (FPG), high density lipoprotein (HDL), triacylglycerol (TAG) and total cholesterol were analysed by the semi automated analyser. Atherogenic index of plasma defined as the base ten logarithm of the ratio of molar concentration of TAG and HDL cholesterol [5]. American Diabetes Association criteria for the diagnosis of DM (FPG $\geq 126 \mathrm{mg} / \mathrm{dl}$ ) and impaired glucose homeostasis (FPG from 100 to $<126 \mathrm{mg}$ / dl) were used. Statistical analysis was done using the SPSS Version 16 statistical package. The probability level was set as $p<0.05$. Initially, possible associations of these factors with DM were determined using univariable analysis. All significant factors identified, were modeled together using binary logistic regression, where presence of DM was the dependent variable.

\section{Results}

A total of 544 participants were selected and the response rate was $95.3 \%(n=511)$. Of them, $224(43.8 \%)$ were male. The overall prevalence of DM was 16.4\% (95\% CI: 13.3-19.9), prevalence was 19.6\% (95\% CI: 14.6-25.4) in males and $13.9 \%$ (95\% CI: 10.1-18.5) in females. Of the participants with diabetes, $27.4 \%$ were previously undiagnosed. Prevalence of pre-diabetes was 7.4\%. Dysglycaemia includes both diabetes and pre-diabetes (FPG $\geq 100 \mathrm{mg} / \mathrm{dl}$ and known diabetics). Total prevalence of dysglycaemia was $23.9 \%$ (95\% CI: 20.2-27.8).

Prevalence of DM in the rural area was 15.2\% (95\% CI: $11.8-19.2)$ and $20.5 \%$ (95\% CI: 13.6-29) in the urban areas. Prevalence of DM was $26.3 \%$ among smokers and $15.2 \%$ among non-smokers. Odds ratio for development of DM among the smokers was significantly higher when compared with non-smokers ( $p=0.032$ ). Prevalence of DM was 21.3\% (95\% CI: 11.9-33.7) among alcohol consumers and $15.8 \%$ (95\% CI: 14.9-23.0) among non-alcohol consumers $(p=0.316)$. Of the participants $29.4 \%$ had a family history of diabetes. A family history of DM was significantly associated with DM (OR=2.95, $p<0.001)$. Prevalence of DM was significantly higher among the participants in the sedentary category (23\%) when compared with the participants in the active category which includes both moderately active and vigorously active participants (13.9\%, $p=0.034)$.

Mean values of biochemical parameters and blood pressure of participants were significantly different among people with diabetes, pre-diabetes and normogycaemic participants $(p<0.05)$, (Table 1$)$. These values were the highest among people with diabetes followed by participants with pre-diabetes.

In the final multivariable model adjusted for age, high WHR and central obesity, participants with family history of diabetes were more likely to develop DM compared to the participants without family history of diabetes (OR 3.5, $p<0.001$ ). Higher WHR carried higher risk (OR 2.0, $p=0.009$ ). Diabetes was not associated with central obesity ( $p=0.00957)$. Risk was higher in older age groups $>65$ years (OR 12.6), 50th years (OR 7.3) and 35-49 years (OR 3-8) compared to 18-34 years age group.

\section{Discussion}

The overall prevalence of DM in the study sample was $16.4 \%$. In a previous study, which covered most areas of the country except the Northern and some parts of Eastern areas, estimated prevalence of DM was 10.3\% [3].

The lowest prevalence of diabetes was observed in the age group of 18-34 (2.2\%) and the highest prevalence was in those aged $>65$ years $(36.4 \%)$. This finding is comparable with the Sri Lanka Diabetes and Cardiovascular Study (1.3\% in age group of 20-29 years, 23.5\% in age group of $\geq 70$ years) [3]. A study conducted in the District of Kalutara has shown that prevalence of DM increased with age up to 59 years and decreased thereafter [6]. Increasing prevalence of diabetes in older persons may be due to lack of exercise, loss of muscle mass, fat deposition and development of insulin resistance as people become older [7].

Odds ratio for developing DM among smokers was significantly higher compared to non-smokers ( $p=0.032$ ). Smoking is an independent risk factor for diabetes, and among diabetics it increases the risk of complications [8]. Smoking has been associated with a higher risk of chronic pancreatitis and pancreatic cancer, suggesting that tobacco smoke may be toxic to the pancreas [9].

Prevalence of DM was significantly higher among sedentary participants (23\%) when compared with active participants which includes both moderately active and vigorously active participants (13.9\%, $p=0.034)$. Physical inactivity is a risk factor for diabetes in Sri Lanka [3]. 
Table 1. Mean values of biochemical parameters and blood pressure of participants with diabetes mellitus, pre-diabetes and normoglycaemic participants

\begin{tabular}{|c|c|c|c|c|c|}
\hline \multirow[t]{2}{*}{ Variables } & \multirow[t]{2}{*}{ Condition } & \multirow[t]{2}{*}{$p$ value } & \multirow[t]{2}{*}{ Mean } & \multicolumn{2}{|c|}{$95 \%$ CI for mean } \\
\hline & & & & Lower bound & Upper bound \\
\hline \multirow{3}{*}{$\begin{array}{l}\text { Fasting plasma } \\
\text { glucose (mg/dL) }\end{array}$} & \multicolumn{2}{|l|}{ Diabetes mellitus } & $143.2( \pm 59.9)$ & 130.2 & 156.2 \\
\hline & Pre-diabetes & 0.000 & $116.2( \pm 3.9)$ & 113.8 & 118.6 \\
\hline & \multicolumn{2}{|l|}{ Normal } & 79.4(土11.7) & 78.3 & 80.5 \\
\hline High density & \multicolumn{2}{|l|}{ Diabetes mellitus } & $35.6( \pm 9.7)$ & 33.5 & 37.7 \\
\hline \multirow[t]{2}{*}{ lipoprotein (mg/dL) } & Pre-diabetes & 0.462 & $33.2( \pm 9.1)$ & 27.7 & 38.7 \\
\hline & \multicolumn{2}{|l|}{ Normal } & $34.3( \pm 9.7)$ & 33.3 & 35.2 \\
\hline \multirow{3}{*}{$\begin{array}{l}\text { Triacylglycerol } \\
\text { (mg/dL) }\end{array}$} & \multicolumn{2}{|l|}{ Diabetes mellitus } & $128.8( \pm 81.1)$ & 111.2 & 146.4 \\
\hline & Pre-diabetes & 0.003 & $96.9( \pm 46.9)$ & 68.6 & 125.2 \\
\hline & \multicolumn{2}{|l|}{ Normal } & $1.0( \pm 64.8)$ & 94.8 & 107.4 \\
\hline \multirow{3}{*}{$\begin{array}{l}\text { Total cholesterol } \\
(\mathrm{mg} / \mathrm{dL})\end{array}$} & \multicolumn{2}{|l|}{ Diabetes mellitus } & $156.0( \pm 43.2)$ & 146.6 & 165.4 \\
\hline & Pre-diabetes & 0.185 & $148.7( \pm 51.4)$ & 117.6 & 179.7 \\
\hline & \multicolumn{2}{|l|}{ Normal } & $147.9( \pm 35.1)$ & 144.5 & 151.3 \\
\hline Atherogenic & \multicolumn{2}{|l|}{ Diabetes mellitus } & $0.23( \pm 0.03)$ & 0.2 & 0.3 \\
\hline \multirow[t]{2}{*}{ index of plasma } & Pre-diabetes & 0.028 & $0.15( \pm 0.07)$ & 0.0 & 0.3 \\
\hline & \multicolumn{2}{|l|}{ Normal } & $0.15( \pm 0.05)$ & 0.1 & 0.2 \\
\hline \multirow{3}{*}{$\begin{array}{l}\text { Systolic blood } \\
\text { pressure (mm Hg) }\end{array}$} & \multicolumn{2}{|l|}{ Diabetes mellitus } & $122( \pm 17)$ & 118.8 & 126.4 \\
\hline & Pre-diabetes & 0.029 & $118( \pm 25)$ & 103.9 & 133.8 \\
\hline & \multicolumn{2}{|l|}{ Normal } & $117( \pm 17)$ & 115.3 & 118.7 \\
\hline \multirow{3}{*}{$\begin{array}{l}\text { Diastolic blood } \\
\text { pressure (mm Hg) }\end{array}$} & \multicolumn{2}{|l|}{ Diabetes mellitus } & $79( \pm 12)$ & 76.5 & 81.6 \\
\hline & Pre-diabetes & 0.238 & $77( \pm 13)$ & 69.0 & 84.9 \\
\hline & \multicolumn{2}{|l|}{ Normal } & $77( \pm 10)$ & 75.8 & 77.9 \\
\hline
\end{tabular}

Financial support: NRC grant 13-122

Family history of diabetes was identified as a risk factor. Family history of diabetes and history of consanguinity have been identified as risk factors for impaired fasting glucose in South East Asians [10]. Asian Indians have strong familial association of DM with a high prevalence of DM among the first degree relatives and vertical transmission through two or more generations [11].

Subjects with high WHR were $(\mathrm{OR}=2,74)$ more likely to develop DM when compared to the subjects with normal WHR. Patients with type 2 DM have higher WHR when compared to non-diabetics [12]. Visceral fat is metabolically active. It releases fatty acids, inflammatory agents, and hormones that lead to higher blood glucose.

The estimated prevalence of DM among adults of Jaffna was $16.4 \%$. Risk of developing DM increased with age. Positive family history of DM and high WHR were other main risk factors for DM in Jaffna district.

\section{References}

1. Venkata C, Ram C, Farmer JA. Metabolic Syndrome in South Asians. J Clin Hypertens 2012; 14: 561-5.

2. Department of Census \& Statistics Preliminary Report, Jaffna District, Department of Census \& Statistics, 2007.

3. Katulanda P, Constantine GR, Mahesh JG, et al. Prevalence and projections of diabetes and pre-diabetes in adults in Sri Lanka - Sri Lanka Diabetes, Cardiovascular Study (SLDCS). Diabet Med 2008; 25: 1062-9.

4. Wijewardene K, Mohideen MR, Mendis S, et al. Prevalence of hypertension, diabetes and obesity: baseline findings of a population based survey in four provinces in Sri Lanka, Ceylon Med J 2005; 50: 62-70.

5. Dobiasova M, Frohlich J, Sedova M, et al. Cholesterol esterification and atherogenic index of plasma correlate with lipoprotein size and findings on coronary angiography. $J$ Lipid Res 2011; 52: 566-71.

6. de Silva AP, de Silva SHP, Liyanage IK, et al. Social, cultural 
and economical determinants of diabetes mellitus in Kalutara district, Sri Lanka: a cross sectional descriptive study. Int $J$ Equity Health 2012; 11: 76.

7. Yumuk VD, Hatemi H, Tarakci T, et al. High prevalence of obesity and diabetes mellitus in Konya, a central Anatolian city in Turkey. Diabetes Res Clin Pr 2005; 70: 151-8.

8. Sairenchi T, Iso H, Nishimura A, et al. Cigarette smoking and risk of type 2 diabetes mellitus among middle-aged and elderly Japanese men and women. Am J Epidemiol 2004; 160: 158-62.

9. Lynch SM, Vrieling A, Lubin JH, et al. Cigarette smoking and pancreatic cancer: a pooled analysis from the pancreatic cancer cohort consortium. Am J Epidemiol 2009; 4: 403-13.

10. Shahid A, Saeed S, Rana S, Mahmood S. Family history of diabetes and parental consanguinity: important risk for impaired fasting glucose in South East Asians, W Indian Med J 2012; 61: 219-23.

11. Ramachandran A, Snehalatha C, Latha E, et al. Impacts of urbanisation on the life style and on the prevalence of diabetes in native Asian Indian population. Diabetes Res Clin Pr 1999; 44: 207-13.

12. Seidell JC, Han TS, Feskens EJM, Lean MEJ. Narrow hips and broad waist circumferences independently contribute to increased risk of non-insulin-dependent diabetes mellitus. J Inter Med 1997; 242: 401-6. 